\title{
Stability Analysis of Grey Discrete Time Time-Delay Systems: A Sufficient Condition
}

\author{
Wen-Jye Shyr ${ }^{1}$ and Chao-Hsing Hsu ${ }^{2}$ \\ ${ }^{1}$ Department of Industrial Education and Technology, \\ National Changhua University of Education \\ 2Department of Computer and Communication Engineering \\ Chienkuo Technology University \\ Changhua 500, Taiwan, \\ R.O.C.
}

\section{Introduction}

Uncertainties in a control system may be the results modeling errors, measurement errors, parameter variations and a linearization approximation. Most physical dynamical systems and industrial process can be described as discrete time uncertain subsystems. Similarly, the unavoidable computation delay may cause a delay time, which can be considered as timedelay in the input part of the original systems. The stability of systems with parameter perturbations must be investigated. The problem of robust stability analysis of a nominally stable system subject to perturbations has attracted wide attention (Mori and Kokame, 1989). Stability analysis attempts to decide whether a system that is pushed slightly from a steadystate will return to that steady state. The robust stability of linear continuous time-delay system has been examined (Su and Hwang, 1992; Liu, 2001). The stability analysis of an interval system is very valuable for the robustness analysis of nominally stable system subject to model perturbations. Therefore, there has been considerable interest in the stability analysis of interval systems (Jiang, 1987; Chou and Chen, 1990; Chen, 1992).

Time-delay is often encountered in various engineering systems, such as the turboject engine, microwave oscillator, nuclear reactor, rolling mill, chemical process, manual control, and long transmission lines in pneumatic and hydraulic systems. It is frequently a source of the generation of oscillation and a source of instability in many control systems. Hence, stability testing for time-delay has received considerable attention (Mori, et al., 1982; Su, et al., 1988; Hmamed, 1991). The time-delay system has been investigated (Mahmoud, et al., 2007; Hassan and Boukas, 2007).

Grey system theory was initiated in the beginning of 1980s (Deng, 1982). Since then the research on theory development and applications is progressing. The state-of-the-art development of grey system theory and its application is addressed (Wevers, 2007). It aims to highlight and analysis the perspective both of grey system theory and of the grey system methods. Grey control problems for the discrete time are also discussed (Zhou and Deng, 1986; Liu and Shyr, 2005). A sufficient condition for the stability of grey discrete time systems with time-delay is proposed in this article. The proposed stability criteria are simple 
to be checked numerically and generalize the systems with uncertainties for the stability of grey discrete time systems with time-delay. Examples are given to compare the proposed method with reported (Zhou and Deng, 1989; Liu, 2001) in Section 4.

The structure of this paper is as follows. In the next section, a problem formulation of grey discrete time system is briefly reviewed. In Section 3, the robust stability for grey discrete time systems with time-delay is derived based on the results given in Section 2. Three examples are given to illustrate the application of result in Section 4 . Finally, Section 5 offers some conclusions.

\section{Problem formulation}

Considering the stability problem of a grey discrete time system is described using the following equation

$$
x(k+1)=A(\otimes) x(k)
$$

where $x(k) \in R^{n}$ represents the state, and $A(\otimes)$ represents the state matrix of system (1). The stability of the system when the elements of $A(\otimes)$ are not known exactly is of major interest. The uncertainty can arise from perturbations in the system parameters because of changes in operating conditions, aging or maintenance-induced errors.

Let $\otimes_{i j}(i, j=1,2, \ldots, n)$ of $A(\otimes)$ cannot be exactly known, but $\otimes_{i j}$ are confined within the intervals $e_{i j} \leq \otimes_{i j} \leq f_{i j}$. These $e_{i j}$ and $f_{i j}$ are known exactly, and $\tilde{\Theta}_{i j} \in[\underline{\otimes}, \bar{\otimes}]$. They are called white numbers, while $\otimes_{i j}$ are called grey numbers. $A(\otimes)$ has a grey matrix, and system (1) is a grey discrete time system.

For convenience of descriptions, the following Definition and Lemmas are introduced.

\section{Definition 2.1}

From system (1), the system has

$$
\begin{aligned}
A(\otimes)=\left[\otimes_{i j}\right]_{n \times n}=\left[\begin{array}{cccc}
\otimes_{11} & \otimes_{12} & \cdots & \otimes_{1 n} \\
\otimes_{21} & \otimes_{22} & \cdots & \otimes_{2 n} \\
\vdots & \vdots & & \vdots \\
\otimes_{n 1} & \otimes_{n 2} & \cdots & \otimes_{n n}
\end{array}\right] \\
E=\left[e_{i j}\right]_{n \times n}=\left[\begin{array}{cccc}
e_{11} & e_{12} & \cdots & e_{1 n} \\
e_{21} & e_{22} & \cdots & e_{2 n} \\
\vdots & \vdots & & \vdots \\
e_{n 1} & e_{n 2} & \cdots & e_{n n}
\end{array}\right] \\
F=\left[f_{i j}\right]_{n \times n}=\left[\begin{array}{cccc}
f_{11} & f_{12} & \cdots & f_{1 n} \\
f_{21} & f_{22} & \cdots & f_{2 n} \\
\vdots & \vdots & & \vdots \\
f_{n 1} & f_{n 2} & \cdots & f_{n n}
\end{array}\right]
\end{aligned}
$$

where $E$ and $F$ represent the minimal and maximal punctual matrices of $A(\otimes)$, respectively. Suppose that $A$ represents the average white matrix of $A(\otimes)$ as 


$$
A=\left[a_{i j}\right]_{n \times n}=\left[\frac{e_{i j}+f_{i j}}{2}\right]_{n \times n}=\frac{E+F}{2}
$$

and

$$
\begin{gathered}
A_{G}=\left[a_{g i j}\right]_{n \times n}=\left[\tilde{\otimes}_{i j}-a_{i j}\right]_{n \times n}=A(\otimes)-A \\
M=\left[m_{i j}\right]_{n \times n}=\left[f_{i j}-a_{i j}\right]_{n \times n}=F-A
\end{gathered}
$$

where $A_{G}$ represents a bias matrix between $A(\otimes)$ and $A ; M$ represents the maximal bias matrix between $F$ and $A$. Then we have

$$
\left|A_{G}\right|_{m} \leq|M|_{m}
$$

where $|M|_{m}$ represents the modulus matrix of $M ; r[M]$ represents the spectral radius of matrix $M$; I represents the identity matrix, and $\lambda(M)$ is the eigenvalue of matrix $M$. This assumption enables some conditions to be derived for the stability of the grey discrete system. Therefore, the following Lemmas are provided.

Lemma 2.1 (Chen, 1984)

The zero state of $x(k+1)=A x(k)$ is asymptotically stable if and only if

$$
|\operatorname{det}(z I-A)|>0, \quad \text { for } \quad|z| \geq 1 \text {. }
$$

Lemma 2.2 (Ortega and Rheinboldt, 1970)

For any $n \times n$ matrices $R, T$ and $V$, if $|R|_{m} \leq V$, then

a. $\quad r[R] \leq r\left[|R|_{m}\right] \leq r[V]$

b. $\quad r[R T] \leq r\left[|R|_{m}|T|_{m}\right] \leq r\left[V|T|_{m}\right]$

c. $\quad r[R+T] \leq r\left[|R+T|_{m}\right] \leq r\left[|R|_{m}+|T|_{m}\right] \leq r\left[V+|T|_{m}\right]$.

Lemma 2.3 (Chou, 1991)

If $G(z)$ is a pulse transfer function matrix, then

$$
|G(z)|_{m} \leq \sum_{k=0}^{\infty}|G(K)|_{m} \equiv H(G(K)), \quad \text { for } \quad|z| \geq 1,
$$

where $G(K)$ is the pulse-response sequence matrix of the multivariable system $G(z)$.

Lemma 2.4 (Chen, 1989)

For an $n \times n$ matrix $R$, if $r[R]<1$, then $|\operatorname{det}(I \pm R)|>0$.

Theorem 2.1

The grey discrete time systems (1) is asymptotically stable, if $A(\otimes)$ is an asymptotically stable matrix, and if the following inequality is satisfied,

$$
r\left[H(G(K))|M|_{m}\right]<1
$$

where $H(G(K))$ and $|M|_{m}$ are defined in Lemma 2.3 and equation (8), and $G(K)$ is the pulseresponse sequence matrix of the system 


\section{Proof}

$$
G(z)=(z I-A)^{-1}
$$

By the identity

$$
\operatorname{det}[R T]=\operatorname{det}[R] \operatorname{det}[T],
$$

for any two $n \times n$ matrices $R$ and $T$, we have

$$
|\operatorname{det}[z I-A(\otimes)]|=\left|\operatorname{det}\left[z I-\left(A+A_{G}\right)\right]\right|=\left|\operatorname{det}\left[I-(z I-A)^{-1}\left(A_{G}\right)\right]\right||\operatorname{det}[z I-A]|
$$

Since $A$ represents an asymptotically stable matrix, then applying Lemma 2.1 clearly shows that

$$
|\operatorname{det}[z I-A]>0|, \text { for }|z| \geq 1
$$

If inequality (9) is satisfied, then Lemmas 2.2 and 2.3 give

$$
\begin{aligned}
r\left[(z I-A)^{-1}\left(A_{G}\right)\right] & =r\left[G(z)\left(A_{G}\right)\right] \leq r\left[|G(z)|_{m}\left|A_{G}\right|_{m}\right] \\
& \leq r\left[|G(z)|_{m}|M|_{m}\right] \\
& \leq r\left[H(G(K))|M|_{m}\right] \\
& <1, \quad \text { for }|z| \geq 1
\end{aligned}
$$

From equations (10)-(12) and Lemma 2.4, we have

$$
\begin{aligned}
|\operatorname{det}[z I-A(\otimes)]| & =\left|\operatorname{det}\left[z I-\left(A+A_{G}\right)\right]\right| \\
& =\left|\operatorname{det}\left[I-(z I-A)^{-1}\left(A_{G}\right)\right]\right| \operatorname{det}[z I-A] \mid>0, \text { for }|z| \geq 1 .
\end{aligned}
$$

Hence, the grey discrete time system (1) is asymptotically stable by Lemma 2.1.

\section{Grey discrete time systems with time-delay}

Considering the grey discrete time system with a time-delay as follows:

$$
x(k+1)=A_{\mathbf{I}}(\otimes) x(k)+B_{\mathbf{I}}(\otimes) x(k-1)
$$

where $A_{\mathbf{I}}(\otimes)$ and $B_{\mathbf{I}}(\otimes)$ denotes interval matrices with the properties as

$$
A_{\mathbf{I}}(\otimes)=\left[\otimes_{i j}^{a}\right]_{n \times n} \text { and } B_{\mathbf{I}}(\otimes)=\left[\otimes_{i j}^{b}\right]_{n \times n}
$$

where $a_{i j}^{1} \leq \otimes_{i j}^{a} \leq a_{i j}^{2}$ and $b_{i j}^{1} \leq \otimes_{i j}^{b} \leq b_{i j}^{2}$.

Indicate

$$
A_{1}=\left[a_{i j}^{1}\right]_{n \times n}, \quad A_{2}=\left[a_{i j}^{2}\right]_{n \times n}, B_{1}=\left[b_{i j}^{1}\right]_{n \times n}, B_{2}=\left[b_{i j}^{2}\right]_{n \times n} .
$$

and let

$$
\mathrm{A}=\left[a_{i j}\right]_{n \times n}=\frac{\left[a_{i j}^{1}+a_{i j}^{2}\right]_{n \times n}}{2}=\frac{\left(A_{1}+A_{2}\right)}{2}
$$


and

$$
\mathrm{B}=\left[b_{i j}\right]_{n \times n}=\frac{\left[b_{i j}^{1}+b_{i j}^{2}\right]_{n \times n}}{2}=\frac{\left(B_{1}+B_{2}\right)}{2}
$$

where $A$ and $B$ are the average matrices between $A_{1}$ and $A_{2}, B_{1}$ and $B_{2}$, respectively. Moreover,

$$
\Delta A_{m}=\left[a_{i j}^{m}\right]_{n \times n}=\left[\otimes_{i j}^{a}-a_{i j}\right]_{n \times n}=A_{I}(\otimes)-A
$$

and

$$
\Delta B_{m}=\left[b_{i j}^{m}\right]_{n \times n}=\left[\otimes_{i j}^{b}-b_{i j}\right]_{n \times n}=B_{I}(\otimes)-B
$$

where $\Delta A_{m}$ and $\Delta B_{m}$ are the bias matrices between $A_{I}$ and $A$, and $B_{I}$ and $B$, respectively. Additionally,

$$
M_{1}=\left[m_{i j}^{1}\right]_{n \times n}=\left[a_{i j}^{2}-a_{i j}\right]_{n \times n}=A_{2}-\mathrm{A}
$$

and

$$
N_{\mathbf{1}}=\left[n_{i j}^{1}\right]_{n \times n}=\left[b_{i j}^{2}-b_{i j}\right]_{n \times n}=B_{\mathbf{2}}-\mathrm{B}
$$

where $M_{1}$ and $N_{1}$ are the maximal bias matrices between $A_{2}$ and $A$, and $B_{2}$ and $B$, respectively. Then we have

$$
\left|\Delta A_{m}\right|_{m} \leq\left|M_{1}\right|_{m} \text { and }\left|\Delta B_{\mathbf{m}}\right|_{m} \leq\left|N_{1}\right|_{m} .
$$

The following theorem ensures the stability of system (13) for all admissible matrices $A, \Delta A_{m}, B$ and $\Delta B_{m}$ with constrained (19).

\section{Theorem 3.1}

The grey discrete time with a time-delay system (13) is asymptotically stable, if nominal system $A_{I}(\otimes)$ is an asymptotically stable matrix, and if the following inequality is satisfied,

$$
r\left[H\left(G_{d}(K)\right)\left(\left|M_{1}\right|_{m}+|B|_{m}+\left|N_{1}\right|_{m}\right)\right]<1
$$

where $H\left(G_{d}(K)\right)$ are as defined in Lemma 2.3, and $G_{d}(K)$ represents the pulse-response sequence matrix of the system

\section{Proof}

$$
G_{d}(z)=(z I-A)^{-1}
$$

By the identity

$$
\operatorname{det}[R T]=\operatorname{det}[R] \operatorname{det}[T],
$$

for any two $n \times n$ matrices $R$ and $T$, we have

$$
\begin{aligned}
& \left|\operatorname{det}\left[z I-\left(A_{I}(\otimes)+B_{I}(\otimes) z^{-1}\right)\right]\right|=\left|\operatorname{det}\left[z I-\left(A+\Delta A_{m}+\left(B+\Delta B_{m}\right) z^{-1}\right)\right]\right| \\
& =\left|\operatorname{det}\left[I-(z I-A)^{-1}\left(\Delta A_{m}+\left(B+\Delta B_{m}\right) z^{-1}\right)\right]\right||\operatorname{det}[z I-A]|
\end{aligned}
$$


Since $A$ is an asymptotically stable matrix, then applying Lemma 2.1 clearly shows that

$$
|\operatorname{det}[z I-A]|>0 \text {, for }|z| \geq 1
$$

If inequality (20) is satisfied, then Lemmas 2.2 and 2.3 give

$$
\begin{aligned}
r\left[(z I-A)^{-1}\left(\Delta A_{m}+\left(B+\Delta B_{m}\right) z^{-1}\right)\right] & =r\left[G_{d}(z)\left(\Delta A_{m}+\left(B+\Delta B_{m}\right) z^{-1}\right)\right] \\
& \leq r\left[\left|G_{d}(z)\right|_{m}\left(\left|\Delta A_{m}+\left(B+\Delta B_{m}\right) z^{-1}\right|_{m}\right)\right] \\
& \leq r\left[\left|G_{d}(z)\right|_{m}\left(\left|\Delta A_{m}\right|_{m}+\left|\left(B+\Delta B_{m}\right) z^{-1}\right|_{m}\right)\right] \\
& \leq r\left[\left|G_{d}(z)\right|_{m}\left(\left|\Delta A_{m}\right|_{m}+\left.\left.\left|\left(B+\Delta B_{m}\right)\right|_{m}\right|^{-1}\right|_{m}\right)\right] \\
& \leq r\left[\left|G_{d}(z)\right|_{m}\left(\left|\Delta A_{m}\right|_{m}+\left|\left(B+\Delta B_{m}\right)\right|_{m}\right)\right] \\
& \leq r\left[\left|G_{d}(z)\right|_{m}\left(\left|\Delta A_{m}\right|_{m}+|B|_{m}+\left|\Delta B_{m}\right|_{m}\right)\right] \\
& \leq r\left[H\left(G_{d}(K)\right)\left(\left|M_{1}\right|_{m}+|B|_{m}+\left|N_{1}\right|_{m}\right)\right] \\
& <1, \text { for }|z| \geq 1
\end{aligned}
$$

Equations (21)-(23) and Lemma 2.4 give

$$
\begin{aligned}
& \left|\operatorname{det}\left[z I-\left(A_{I}(\otimes)+B_{I}(\otimes) z^{-1}\right)\right]\right|=\left|\operatorname{det}\left[z I-\left(A+\Delta A_{m}+\left(B+\Delta B_{m}\right) z^{-1}\right)\right]\right| \\
& \quad=\left|\operatorname{det}\left[I-(z I-A)^{-1}\left(\Delta A_{m}+\left(B+\Delta B_{m}\right) z^{-1}\right)\right]\right||\operatorname{det}[z I-A]|>0, \text { for }|z| \geq 1
\end{aligned}
$$

Therefore, by Lemma 2.1, the grey discrete time with a time-delay system (13) is asymptotically stable.

\section{4. llustrative examples}

\section{Example 4.1}

Consider the stability of grey discrete time system (1) as follows:

$$
x(k+1)=A(\otimes) x(k),
$$

where

$$
A(\otimes)=\left[\begin{array}{ll}
\otimes_{11}^{a} & \otimes_{12}^{a} \\
\otimes_{21}^{a} & \otimes_{22}^{a}
\end{array}\right]
$$

with $-0.5 \leq \otimes_{11}^{a} \leq 0.5,0.1 \leq \otimes_{12}^{a} \leq 0.8,-0.3 \leq \otimes_{21}^{a} \leq 0.2,-0.4 \leq \otimes_{22}^{a} \leq 0.5$.

From equations (2)-(5), the average matrices is

$$
A=\left[\begin{array}{cc}
0 & 0.45 \\
-0.05 & 0.05
\end{array}\right],
$$


and from equations (6)-(7), the maximal bias matrix $M$ is

$$
\mathrm{M}=\left[\begin{array}{cc}
0.5 & 0.35 \\
0.25 & 0.45
\end{array}\right]
$$

By Lemma 2.3, we obtain

$$
\mathrm{H}(\mathrm{G}(\mathrm{K}))=\left[\begin{array}{ll}
1.0241 & 0.4826 \\
0.0536 & 1.0725
\end{array}\right]
$$

Then, the equation (9) is

$$
r\left[H(G(K))|M|_{m}\right]=0.9843<1 .
$$

Therefore, the system (1) is asymptotically stable in terms of Theorem 2.1.

\section{Remark 1}

Zhou and Deng (1989) have illustrated that the grey discrete time system (1) is asymptotically stable if the following inequality holds:

$$
\rho(k)<1
$$

By applying the condition (24) as given by Zhou and Deng, the sufficient condition can be obtained as $\rho(k)=0.9899<1$ to guarantee that the system (1) is still stable.

The proposed sufficient condition (9) of Theorem 2.1 is less conservative than the condition (24) proposed by Zhou and Deng.

\section{Example 4.2}

Considering the grey discrete time with a time-delay system (Shyr and Hsu, 2008) is described by (13) as follows:

$$
x(k+1)=A_{\mathbf{I}}(\otimes) x(k)+B_{\mathbf{I}}(\otimes) x(k-1)
$$

where

$$
A_{I}(\otimes)=\left[\begin{array}{ll}
\otimes_{11}^{a} & \otimes_{12}^{a} \\
\otimes_{21}^{a} & \otimes_{22}^{a}
\end{array}\right], B_{I}(\otimes)=\left[\begin{array}{ll}
\otimes_{11}^{b} & \otimes_{12}^{b} \\
\otimes_{21}^{b} & \otimes_{22}^{b}
\end{array}\right],
$$

with

$$
-0.2 \leq \otimes_{11}^{a} \leq 0.2,-0.2 \leq \otimes_{12}^{a} \leq 0.1,-0.1 \leq \otimes_{21}^{a} \leq 0.1,-0.1 \leq \otimes_{22}^{a} \leq 0.2
$$

and

$$
0.1 \leq \otimes_{11}^{b} \leq 0.2,0.1 \leq \otimes_{12}^{b} \leq 0.2,0.1 \leq \otimes_{21}^{b} \leq 0.15,0.2 \leq \otimes_{22}^{b} \leq 0.25 .
$$

Equation (15) and (25) give

$$
A_{1}=\left[\begin{array}{cc}
-0.2 & -0.2 \\
-0.1 & -0.1
\end{array}\right], \quad A_{2}=\left[\begin{array}{ll}
0.2 & 0.1 \\
0.1 & 0.2
\end{array}\right], \quad B_{1}=\left[\begin{array}{ll}
0.1 & 0.1 \\
0.1 & 0.2
\end{array}\right], \quad B_{2}=\left[\begin{array}{cc}
0.2 & 0.2 \\
0.15 & 0.25
\end{array}\right]
$$


From equations (16), the average matrices are

$$
A=\left[\begin{array}{cc}
0 & -0.05 \\
0 & 0.05
\end{array}\right], \quad B=\left[\begin{array}{cc}
0.15 & 0.15 \\
0.125 & 0.225
\end{array}\right],
$$

and from equations (18), the maximal bias matrices $M_{1}$ and $N_{1}$ are

$$
M_{1}=\left[\begin{array}{ll}
0.2 & 0.15 \\
0.1 & 0.15
\end{array}\right], \quad N_{1}=\left[\begin{array}{ll}
0.05 & 0.05 \\
0.025 & 0.025
\end{array}\right] .
$$

By Lemma 2.3, we obtain

$$
\mathrm{H}\left(\mathrm{G}_{\mathrm{d}}(\mathrm{K})\right)=\left[\begin{array}{cc}
0.0526 & 0.0526 \\
0 & 1.0526
\end{array}\right]
$$

From Theorem 3.1, the system (13) is stable, because

$$
r\left[H\left(G_{d}(K)\right)\left(\left|M_{1}\right|_{m}+|B|_{m}+\left|N_{\mathbf{1}}\right|_{m}\right)\right]=0.4462<1 .
$$

\section{Example 4.3}

Considering the grey discrete time-delay systems (Zhou and Deng, 1989) is described by (13), where

$$
A_{I}(\otimes)=\left[\begin{array}{ll}
\otimes_{11}^{a} & \otimes_{12}^{a} \\
\otimes_{21}^{a} & \otimes_{22}^{a}
\end{array}\right], B_{I}(\otimes)=\left[\begin{array}{ll}
\otimes_{11}^{b} & \otimes_{12}^{b} \\
\otimes_{21}^{b} & \otimes_{22}^{b}
\end{array}\right],
$$

with $-0.24 \leq \otimes_{11}^{a} \leq 0.24,0.12 \leq \otimes_{12}^{a} \leq 0.24,-0.12 \leq \otimes_{21}^{a} \leq 0.12,0.12 \leq \otimes_{22}^{a} \leq 0.24$ and

$$
0.12 \leq \otimes_{11}^{b} \leq 0.24,0.12 \leq \otimes_{12}^{b} \leq 0.24,0.12 \leq \otimes_{21}^{b} \leq 0.18,0.24 \leq \otimes_{22}^{b} \leq 0.30 .
$$

Equation (15) and (25) give

$$
A_{1}=\left[\begin{array}{ll}
-0.24 & 0.12 \\
-0.12 & 0.12
\end{array}\right], A_{2}=\left[\begin{array}{ll}
0.24 & 0.24 \\
0.12 & 0.24
\end{array}\right], B_{1}=\left[\begin{array}{ll}
0.12 & 0.12 \\
0.12 & 0.24
\end{array}\right], B_{2}=\left[\begin{array}{ll}
0.24 & 0.24 \\
0.18 & 0.30
\end{array}\right] .
$$

From (16)-(18), we obtain the matrices

$$
A=\left[\begin{array}{ll}
0 & 0.18 \\
0 & 0.18
\end{array}\right], M_{1}=\left[\begin{array}{ll}
0.24 & 0.06 \\
0.12 & 0.06
\end{array}\right], B=\left[\begin{array}{ll}
0.18 & 0.18 \\
0.15 & 0.27
\end{array}\right], N_{\mathbf{1}}=\left[\begin{array}{ll}
0.06 & 0.06 \\
0.03 & 0.03
\end{array}\right]
$$

By Lemma 2.3, we obtain

$$
\mathrm{H}\left(\mathrm{G}_{\mathrm{d}}(\mathrm{K})\right)=\left[\begin{array}{ll}
0.5459 & 0.3790 \\
0.3659 & 0.4390
\end{array}\right] .
$$

From Theorem 3.1, the system (13) is stable, because

$$
r\left[H\left(G_{d}(K)\right)\left(\left|M_{1}\right|_{m}+|B|_{m}+\left|N_{1}\right|_{m}\right)\right]=0.8686<1
$$


According to Theorem 3.1, we know that system (13) is asymptotically stable.

\section{Remark 2}

If the following condition holds (Liu, 2001)

$$
\min \left\{\max _{i} \sum_{j=1}^{n}\left(e_{i j}+f_{i j}\right), \max _{i} \sum_{j=1}^{n}\left(e_{j i}+f_{j i}\right)\right\}<1
$$

then system (13) is stable $i, j=1,2, \ldots, n$, where

$$
E=\left[e_{i j}\right], e_{i i}=a_{i j}^{2}, \quad e_{i j}=\max \left\{\left|\otimes_{i j}^{a}\right|,\left|a_{i j}^{2}\right|\right\} \quad \text { for } i \neq j
$$

and

$$
F=\left[f_{i j}\right], f_{i i}=b_{i j}^{2}, f_{i j}=\max \left\{\left|\otimes_{i j}^{a}\right|,\left|b_{i j}^{2}\right|\right\} \quad \text { for } i \neq j
$$

The foregoing criterion is applied in our example and we obtain

$$
\min \left\{\max _{i} \sum_{j=1}^{n}\left(e_{i j}+f_{i j}\right), \max _{i} \sum_{j=1}^{n}\left(e_{j i}+f_{j i}\right)\right\}=1.02>1
$$

which cannot be satisfied in (26).

\section{Conclusions}

This paper proposes a sufficient condition for the stability analysis of grey discrete time systems with time-delay whose state matrices are interval matrices. A novel sufficient condition is obtained to ensure the stability of grey discrete time systems with time-delay. By mathematical analysis, the stability criterion of the proposed is less conservative than those of previous results. In Remark 1, by mathematical analysis, the presented criterion is less conservative than that proposed by Zhou and Deng (1989). In Remarks 2, by mathematical analysis, the presented criterion is to be less conservative than that proposed by Liu (2001). Therefore, the results of this paper indeed provide an additional choice for the stability examination of the grey discrete time time-delay systems. The proposed examples clearly demonstrate that the criteria presented in this paper for the stability of grey discrete time systems with time-delay are useful.

\section{References}

Chen, C. T. (1984). Linear system theory and design, New York: Pond Woods, Stony Brook.

Chen, J. (1992). Sufficient conditions on stability of interval matrices: connections and new results, IEEE Transactions on Automatic Control, Vol.37, No.4, pp.541-544.

Chen, K. H. (1989). Robust analysis and design of multi-loop control systems, Ph. D. Dissertation National Tsinghua University, Taiwan, R.O.C.

Chou, J. H. (1991). Pole-assignment robustness in a specified disk, Systems Control Letters, Vol.6, pp.41-44. 
Chou, J. H. and Chen, B. S. (1990). New approach for the stability analysis of interval matrices, Control Theory and Advanced Technology, Vol.6, No.4, pp.725-730.

Deng, J. L. (1982). Control problem of grey systems, Systems \& Control Letters, Vol.1, No.5, pp.288-294.

Hassan, M. F. and Boukas, K. (2007). Multilevel technique for large scale LQR with timedelays and systems constraints, International Journal of Innovative Computing Information and Control, Vol.3, No.2, pp.419-434.

Hmamed, A. (1991). Further results on the stability of uncertain time-delay systems, International Journal of Systems Science, Vol.22, pp.605-614.

Jiang, C. L. (1987). Sufficient condition for the asymptotic stability of interval matrices, International Journal of Control, Vol.46, No.5, pp.1803.

Liu, P. L. (2001). Stability of grey continuous and discrete time-delay systems, International Journal of Systems Science, Vol.32, No.7, pp.947-952.

Liu, P. L. and Shyr, W. J. (2005). Another sufficient condition for the stability of grey discrete-time systems, Journal of the Franklin Institute-Engineering and Applied Mathematics, Vol.342, No.1, pp.15-23.

Lu, M. and Wevers, K. (2007). Grey system theory and applications: A way forward, Journal of Grey System, Vol.10, No.1, pp.47-53.

Mahmoud, M. S., Shi, Y. and Nounou, H. N. (2007). Resilient observer-based control of uncertain time-delay systems, International Journal of Innovative Computing Information and Control, Vol.3, No.2, pp. 407-418.

Mori, T. and Kokame, H. (1989). Stability of $\dot{x}(t)=A x(t)+B x(t-\tau)$, IEEE Transactions on Automatic Control, Vol. 34, No.1, pp.460-462.

Mori, T., Fukuma, N. and Kuwahara, M. (1982). Delay-independent stability criteria for discrete-delay systems, IEEE Transactions on Automatic Control, Vol.27, No.4, pp.964-966.

Ortega, J. M. and Rheinboldt, W. C. (1970). Interactive soluation of non-linear equation in several variables, New York : Academic press.

Shyr W. J. and Hsu, C. H. (2008). A sufficient condition for stability analysis of grey discretetime systems with time delay, International Journal of Innovative Computing Information and Control, Vol.4, No.9, pp.2139-2145.

$\mathrm{Su}, \mathrm{T}$. J. and Hwang, C. G. (1992). Robust stability of delay dependence for linear uncertain systems, IEEE Transactions on Automatic Control, Vol.37, No.10, pp.1656-1659.

Su, T. J., Kuo, T. S. and Sun, Y. Y. (1988). Robust stability for linear time-delay systems with linear parameter perturbations, International Journal of Systems Science, Vol.19, pp.2123-2129.

Zhou, C. S. and Deng, J. L. (1986). The stability of the grey linear system, International Journal of Control, Vol. 43, pp.313-320.

Zhou, C. S. and Deng, J. L. (1989). Stability analysis of grey discrete-time systems, IEEE Transactions on Automatic Control, Vol.34, No.2, pp.173-175. 


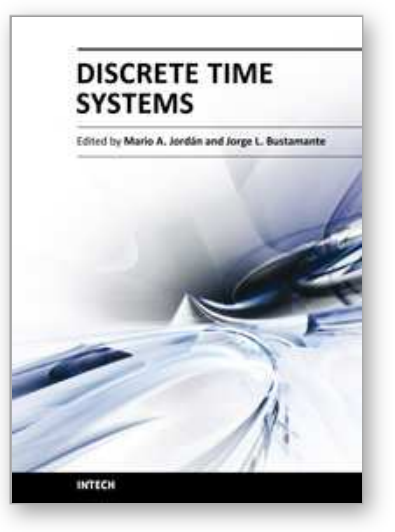

\author{
Discrete Time Systems \\ Edited by Dr. Mario Alberto Jord $\tilde{A}_{i n}$
}

ISBN 978-953-307-200-5

Hard cover, 526 pages

Publisher InTech

Published online 26, April, 2011

Published in print edition April, 2011

Discrete-Time Systems comprehend an important and broad research field. The consolidation of digital-based computational means in the present, pushes a technological tool into the field with a tremendous impact in areas like Control, Signal Processing, Communications, System Modelling and related Applications. This book attempts to give a scope in the wide area of Discrete-Time Systems. Their contents are grouped conveniently in sections according to significant areas, namely Filtering, Fixed and Adaptive Control Systems, Stability Problems and Miscellaneous Applications. We think that the contribution of the book enlarges the field of the Discrete-Time Systems with signification in the present state-of-the-art. Despite the vertiginous advance in the field, we also believe that the topics described here allow us also to look through some main tendencies in the next years in the research area.

\title{
How to reference
}

In order to correctly reference this scholarly work, feel free to copy and paste the following:

Wen-Jye Shyr and Chao-Hsing Hsu (2011). Stability Analysis of Grey Discrete Time Time-Delay Systems: A Sufficient Condition, Discrete Time Systems, Dr. Mario Alberto JordÃ $i n$ (Ed.), ISBN: 978-953-307-200-5, InTech, Available from: http://www.intechopen.com/books/discrete-time-systems/stability-analysis-of-greydiscrete-time-time-delay-systems-a-sufficient-condition

\section{INTECH}

open science | open minds

\author{
InTech Europe \\ University Campus STeP Ri \\ Slavka Krautzeka 83/A \\ 51000 Rijeka, Croatia \\ Phone: +385 (51) 770447 \\ Fax: +385 (51) 686166 \\ www.intechopen.com
}

\author{
InTech China \\ Unit 405, Office Block, Hotel Equatorial Shanghai \\ No.65, Yan An Road (West), Shanghai, 200040, China \\ 中国上海市延安西路65号上海国际贵都大饭店办公楼 405 单元 \\ Phone: +86-21-62489820 \\ Fax: $+86-21-62489821$
}


(C) 2011 The Author(s). Licensee IntechOpen. This chapter is distributed under the terms of the Creative Commons Attribution-NonCommercialShareAlike-3.0 License, which permits use, distribution and reproduction for non-commercial purposes, provided the original is properly cited and derivative works building on this content are distributed under the same license. 